\title{
Avaliação da evolução pós-operatória dos ependimomas intramedulares
}

\author{
Marcos Juliano dos Santos', Enrico Guinzoni', Andrei Fernandes Joaquim ${ }^{1}$, Helder Tedeschi \\ Faculdade de Ciências Médicas da Universidade Estadual de Campinas (FCM/Unicamp), Campinas, SP, Brasil.
}

\section{RESUMO}

Objetivo: Avaliar a eficácia da técnica microcirúrgica utilizada para ressecção completa dos ependimomas intramedulares sem agregar déficits aos pacientes, em especial, aos pacientes funcionalmente independentes no pré-operatório. Método: Este estudo avaliou, segundo a escala funcional de McCormick, a evolução pós-operatória de uma série de 20 pacientes submetidos à ressecção microcirúrgica total para ependimomas intramedulares. Resultados: A ressecção completa foi atingida em 19 dos 20 pacientes (95\%) e somente um paciente apresentou piora clínica (5\%). Nos pacientes com independência funcional pré-operatória, com McCormick grau I e II, não houve piora clínica e todos os tumores foram ressecados completamente. No subgrupo de pacientes grau II, a média do status funcional pós-operatória apresentou melhora estatisticamente significativa. Nenhum paciente com grau IV melhorou após o tratamento cirúrgico. Conclusão: O tratamento cirúrgico foi eficaz para ressecar completamente os tumores sem agregar déficits neurológicos na maioria dos pacientes. Nos pacientes com McCormick graus I e II pré-operatórios a cirurgia deve ser indicada no momento do diagnóstico.

\section{PALAVRAS-CHAVE}

Ependimomas, neoplasias da medula espinal, microcirurgia.

\begin{abstract}
Post-operative outcome evaluation of intramedullary ependymomas

Objective: Evaluate the efficacy of the microsurgery technique used aiming total tumor resection without causing neurological deficits, with special attention to the patients with good functional status before surgery. Method: This study has evaluated according to the McCormick functional scale the surgical outcome of a series of twenty patients with intramedullary ependymomas submitted to microsurgical resection. Results: Total surgical resection was achieved in 19 of the 20 patients (95\%). Only one patient experienced clinical worsening (5\%). Patients classified as McCormick grade I and II who were independent pre-operatively remained so in the post-operative period and had their tumors completely removed. In grade II patients there was a significant improvement in their post-operative status. None of the grade IV patients improved after surgical treatment. Conclusion: Surgical treatment has proven to be efficient in completely removing tumors without adding neurological deficits in most patients. In patients with McCormick grades I and II pre-operatively surgery should be indicated early in the diagnosis.
\end{abstract}

\section{KEYWORDS}

Ependymomas, spinal cord neoplasms, microsurgery.

\section{Introdução}

A primeira ressecção de tumor intramedular publicada na literatura médica deve-se a Elsberg em 1911. ${ }^{1}$ Em virtude da morbidade dos procedimentos para patologias medulares intrínsecas e sua dificuldade de diagnóstico, a remoção cirúrgica de tais lesões permaneceu em segundo plano nas opções de tratamento existentes até a década de 1980. A partir de então, o avanço das técnicas microcirúrgicas e da microscopia e o surgimento da mielotomografia e da ressonância magnética possibilitaram um diagnóstico mais preciso e a remoção cirúrgica mais segura. ${ }^{2,3}$

O ependimoma é o tumor intramedular mais frequente na idade adulta, correspondendo a $45 \%-50 \%$ dos casos, mas é raro (apenas 1\%) quando comparado aos tumores do sistema nervoso central como um todo. ${ }^{3-7}$

A grande maioria dos ependimomas intramedulares é constituída por tumores benignos, de crescimento lento, ${ }^{6}$ grau II pela Organização Mundial da Saúde (OMS)

1 Neurocirurgião, pós-graduando da Faculdade de Ciências Médicas da Universidade Estadual de Campinas (FCM/Unicamp), Campinas, SP, Brasil.

2 Neurocirurgião, professor doutor da FCM/Unicamp, Campinas, SP, Brasil. 
e que acometem uma população de adultos jovens predominantemente. Sua história natural é caracterizada inicialmente por sintomas sensitivos, seguidos de déficits motores e esfincterianos, os quais se mostram progressivamente limitantes. Por essas características, é uma entidade patológica cuja remoção cirúrgica tem impacto decisivo na evolução dos pacientes, podendo potencialmente determinar a cura dos indivíduos acometidos e prevenir a instalação de quadros deficitários permanentes.

Apresentamos a técnica microcirúrgica utilizada em nosso serviço para ressecção dos ependimomas intramedulares e seus resultados.

\section{Método}

Entre janeiro de 2000 e novembro de 2011, 27 pacientes com ependimomas espinais foram submetidos à ressecção microcirúrgica pela equipe da FCM-Unicamp; desses, 20 foram analisados em nosso estudo. Os fatores de exclusão foram: localização do tumor na cauda equina ou no filo terminal; pacientes submetidos a tratamento radioterápico ou quimioterápico prévios; pacientes portadores de outras patologias neurológicas que reconhecidamente pudessem contribuir com déficits neurológicos como acidentes vasculares cerebrais, esclerose múltipla e mielopatia espondilótica e pacientes com dados incompletos ou imprecisos.
O status funcional clínico foi medido segundo a escala de McCormick e Stein ${ }^{8}$ (Quadro 1) no pré-operatório e no pós-operatório tardio de seis meses. Todos os pacientes tiveram a hipótese diagnóstica e o grau de ressecção estabelecidos por ressonância magnética pré e pós-operatória (Figura 1). Tal exame foi repetido anualmente no seguimento para cada paciente.

Todos os dados foram colhidos de modo retrospectivo pela análise dos prontuários.

\section{Técnica cirúrgica}

Os pacientes foram submetidos a laminotomia ou laminectomia com o auxílio de radioscopia nos níveis

\begin{tabular}{|l|}
\hline \multicolumn{1}{|c|}{ Quadro 1 - Escala funcional de McCormick } \\
\hline Escala de McCormick (McCormick et al., 1990) \\
\hline Grau: \\
• I: Neurologicamente normal; déficit leve, não \\
afetando a função significativamente; alteração de \\
reflexo ou espasticidade; marcha normal \\
- II: Déficit sensitivo ou motor, afetando a função em \\
um membro; déficit leve a moderado na marcha; \\
disestesia grave; deambula independentemente \\
- III: Déficit grave; ajuda ou suporte para deambular; \\
pode ou não estar funcionalmente dependente \\
IV: Déficit gravíssimo; em cadeira de rodas com déficit bilateral \\
grave em membros superiores; geralmente dependente
\end{tabular}

McCormick PC, Stein BM. Intramedullary tumors in adults. Neurosurg Clin N Am. 1990;1(3):609-30.

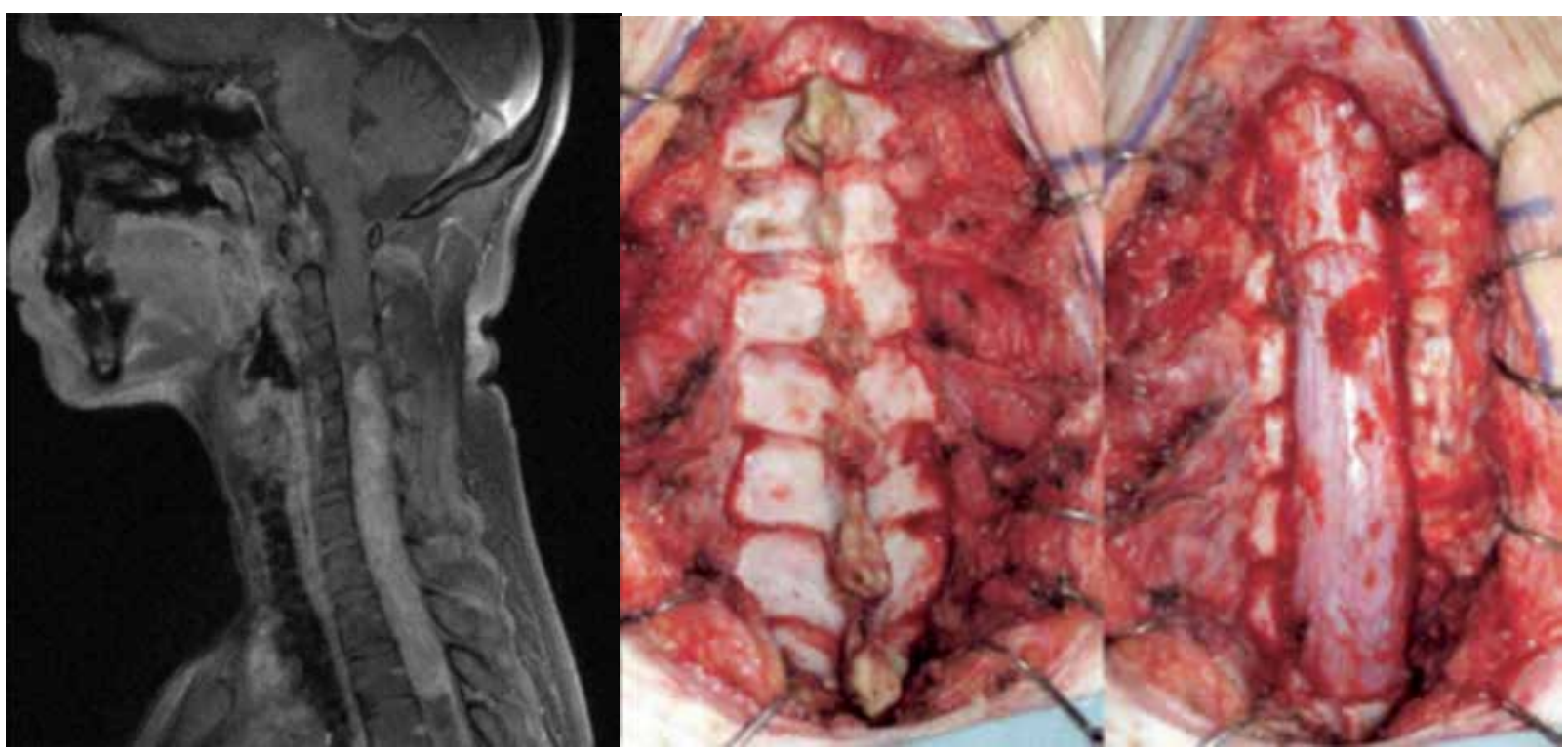

Figura 1 - Aspecto típico dos ependimomas intramedulares na ressonância magnética e laminotomia.

Da esquerda para direita: Aspecto radiológico típico de ependimoma intramedular, com polos captantes de contraste bem delimitados e edema medular adjacente. Exposição das lâminas cervicais após dissecção subperiosteal da musculatura paravertebral. Laminotomia em open door retraída com anzóis. 
acometidos e em um nível cranial e um nível caudal aos limites da lesão estabelecidos pela ressonância magnética (Figura 1). A dura-máter foi aberta com incisão mediana ao longo de toda sua exposição e suturas de retração lateral foram aplicadas às suas margens. Depois de adequada exposição da face dorsal da medula, uma mielotomia é iniciada no ponto médio da lesão em relação a seus limites superior e inferior (Figura 2). A linha

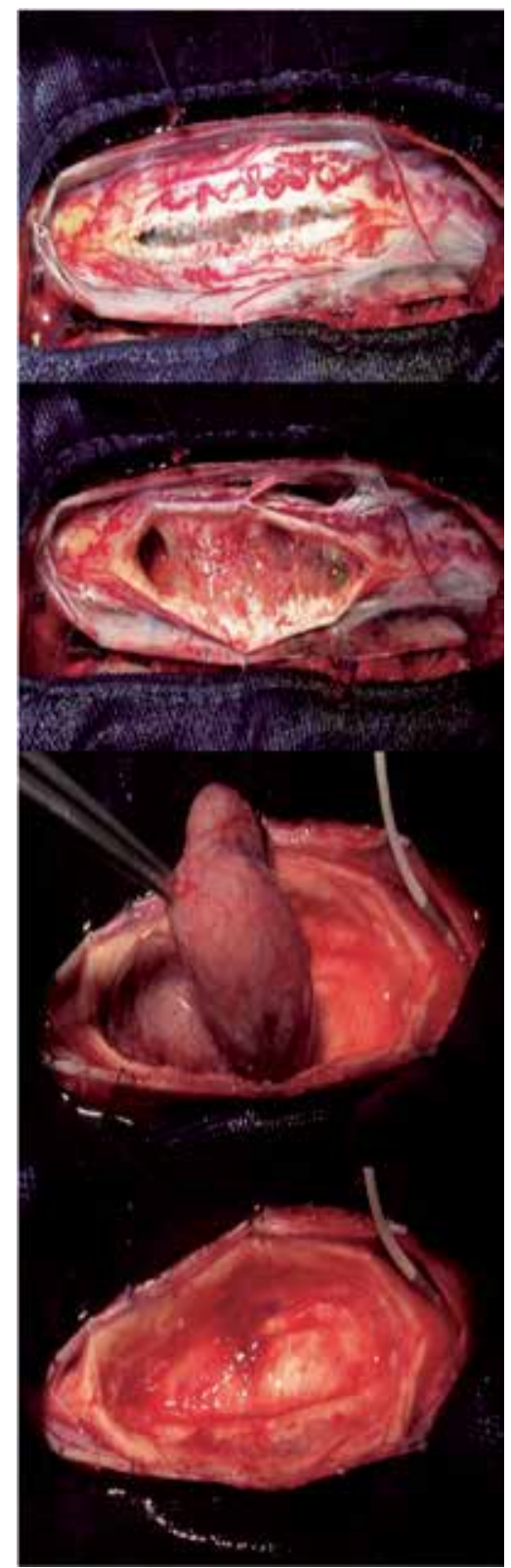

Figura 2 - Sequência para ressecção microcirúrgica em bloco de ependimoma intramedular.

De cima para baixo. A mielotomia é realizada no ponto equidistante entre a entrada das raizes sensitivas e realizada até as extremidades da lesão. Pontos de retração pial são realizados para evitar pressão dos instrumentos cirúrgicos sobre a medula. Após dissecção periférica da lesão, o polo inferior é tracionado para dissecção da face ventral da lesão. Aspecto cirúrgico após retirada em bloco da lesão. média é o plano sagital adequado e é assegurada pelo ponto de entrada dos vasos na rafe mediana posterior e pelo ponto equidistante da saída das raízes dorsais. Após coagulação bipolar em baixa voltagem, realiza-se a abertura da pia-máter com lâmina 11 e procede-se à dissecção romba com microdissectores até encontrar-se a superfície tumoral. A partir do ponto médio da lesão a mielotomia é estendida até os polos cranial e caudal do tumor (Figura 2). Pontos de sutura são empregados para retração lateral na pia-máter. A retração da pia-máter é essencial na dissecção do plano de clivagem entre o tumor e o tecido neural, pois permite que a tração seja aplicada pelo cirurgião somente sobre o tumor, o que diminui a manipulação de tecido medular. Os vasos que atravessam os limites entre o tecido neural e o tumor são coagulados com bipolar e seccionados progressivamente determinando um plano de separação lateralmente. A separação da face ventral da lesão da rafe, dos funículos anteriores e da artéria espinhal anterior representa a maior dificuldade técnica do procedimento. É principalmente nessa fase que déficits permanentes podem ser estabelecidos. Quanto maior o diâmetro axial da lesão, mais íntima é a sua relação com a artéria espinhal anterior e maior a aderência da lesão com a pia-máter do sulco mediano anterior. Após a soltura do polo inferior ou superior do tumor, este é tracionado no sentido oposto, para que tal separação ocorra meticulosamente até a liberação completa da lesão e sua retirada em bloco (Figura 2). Não foi utilizado aspirador ultrassônico na ressecção dos tumores nesta série. Somente seis dos 20 pacientes foram submetidos à monitorização de potenciais evocados sensitivos e motores.

\section{Resultados}

Vinte pacientes foram submetidos à ressecção cirúrgica de ependimomas intramedulares, com a duração média dos sintomas de 17 meses (3-36 meses). A média de idade foi de 33,75 anos, variando de 13 a 55 anos. Quanto a sua localização, dois tumores eram na transição crânio-cervical (10\%), seis eram cervicais (30\%), quatro, cervicotorácicos (20\%) e oito torácicos (40\%). Não houve diferença na distribuição dos casos entre sexos. A tabela 1 mostra a distribuição dos casos em relação à classificação clínica funcional pela escala de McCormick no pré e pós-operatório tardio $(6 \mathrm{~m})$. No pré-operatório, a distribuição foi de 7 (35\%) pacientes de grau I, 7 (35\%) de grau II, 4 (20\%) de grau III e 2 (10\%) de grau IV. Dos 20 pacientes analisados, 5 (25\%) melhoraram seu status funcional após o tratamento cirúrgico, 15 (70\%) permaneceram clinicamente inalterados e 1 (5\%) piorou. A ressecção completa ocorreu em 19/20 pacientes, correspondendo 
a $95 \%$ dos casos. O paciente que teve o tumor ressecado de modo subtotal foi o único que apresentou caráter infiltrativo no intraoperatório, e o anatomopatológico demonstrou ependimoma anaplásico OMS III, todos os 19 pacientes restantes tiveram ressecção completa pela ressonância magnética pós-operatória e apresentaram histologia benigna OMS grau II.

O período de follow-up médio foi de 4,57 anos por paciente, variando de 1 a 11 anos. Não houve recidivas das lesões ressecadas totalmente no período avaliado. O paciente que apresentou ependimoma anaplásico e ressecção parcial apresentou crescimento da lesão, foi reabordado e é o único da série a ser submetido à radioterapia, mas faleceu de complicações clínicas após um ano de seguimento.

O McCormick médio nos 20 pacientes no pré-operatório foi de 2,05, sendo de 1,85 no pós-operatório tardio (maior de seis meses). Essa diferença não foi estatisticamente significativa no teste T, $\operatorname{com} \mathrm{p}=0,0518$. Analisando somente os pacientes classificados como McCormick II no pré-operatório, a média no pré-operatório de 2 mudou para 1,57 no pós-operatório tardio, no teste $\mathrm{T}$, e essa diferença foi significativa, $\operatorname{com} p=0,039(p<0,05)$.

Entre os pacientes com McCormick grau I pré-operatório, nenhum paciente piorou seu status clínico, e todos tiveram seus tumores ressecados completamente. Nenhum paciente com grau IV pré-operatório melhorou. Dos quatro pacientes com grau III antes do tratamento, dois melhoraram, um piorou e um permaneceu inalterado. A média do grau funcional no pós-operatório foi de 1,85 , no entanto essa melhora não foi estatisticamente significativa, com $\mathrm{p}=0,31$.

Não houve infecção de ferida operatória ou fístula liquórica nesta série. Dois pacientes apresentaram cifotização na transição cervicotorácica na evolução em longo prazo e foram submetidos à artrodese cervicotorácica posteriormente. Houve um óbito durante o seguimento no paciente que apresentou ependimoma anaplásico aos 12 meses de pós-operatório com recidivas em todo neuroeixo, inclusive cerebrais.

\section{Discussão}

Os ependimomas intramedulares são lesões de evolução lenta que progressivamente levam a déficits neurológicos limitantes. A ressonância magnética é diagnóstica da lesão, com especificidade de $95 \%{ }^{3}$ A grande maioria dos tumores é de histologia benigna

\begin{tabular}{|c|c|c|c|c|c|}
\hline Caso & Idade & Topografia & $\begin{array}{l}\text { Escala funcional } \\
\text { de McCormick - } \\
\text { Pré-operatório }\end{array}$ & $\begin{array}{l}\text { Escala funcional } \\
\text { de McCormick - } \\
\text { Pós-operatório }\end{array}$ & Complicações \\
\hline 1 & 31 & $\mathrm{C}$ & II & I & \\
\hline 2 & 31 & CT & II & II & Cifose \\
\hline 3 & 29 & $\mathrm{~T}$ & I & I & \\
\hline 4 & 44 & $\mathrm{C}$ & II & I & \\
\hline 5 & 37 & CT & II & II & Cifose \\
\hline 6 & 51 & $\mathrm{~T}$ & I & I & \\
\hline 7 & 55 & $\mathrm{~T}$ & II & II & \\
\hline 8 & 34 & $\mathrm{CB}$ & III & II & \\
\hline 9 & 33 & $\mathrm{CB}$ & II & I & \\
\hline 10 & 33 & $\mathrm{~T}$ & II & II & \\
\hline 11 & 30 & $\mathrm{~T}$ & I & I & \\
\hline 12 & 31 & $\mathrm{C}$ & I & I & \\
\hline 13 & 31 & $\mathrm{~T}$ & III & IV & Piora clínica \\
\hline 14 & 35 & $\mathrm{~T}$ & I & I & \\
\hline 15 & 24 & $\mathrm{C}$ & I & I & \\
\hline 16 & 40 & $\mathrm{C}$ & I & I & \\
\hline 17 & 30 & $\mathrm{CT}$ & III & II & \\
\hline 18 & 13 & $\mathrm{C}$ & IV & IV & \\
\hline 19 & 29 & $\mathrm{~T}$ & IV & IV & \\
\hline 20 & 34 & $\mathrm{CT}$ & III & III & \\
\hline
\end{tabular}

CB: bulco-cervical; C: cervical; CT: cervicotorácico, T: torácico. 
da variante celular grau II da OMS. O principal fator prognóstico é o grau funcional do paciente antes do tratamento cirúrgico ${ }^{7-13}$ quanto menor o grau funcional de McCormick, melhores as chances de resultado cirúrgico satisfatório, ou seja, ressecção total sem piora clínica.

Apesar de a ressecção total da lesão ser o objetivo principal desde as séries publicadas por Epstein et al. ${ }^{3}$ e McCormick e Stein, ${ }^{8}$ diferentes estratégias microcirúrgicas têm sido utilizadas para atingir esse objetivo.

A dissecção periférica inicial seguida de esvaziamento interno, ou debulking, da lesão é a técnica descrita por Epstein et al. ${ }^{3}$ e utilizada nos últimos anos por outros autores. ${ }^{10,13}$ Ela consiste no esvaziamento interno da lesão, geralmente com a utilização de aspirador ultrassônico, para posterior separação da superfície tumoral restante do tecido medular.

Nesta série aplicamos a técnica de delimitação microcirúrgica de toda a periferia da lesão sem esvaziamento interno, e os ependimomas foram ressecados em bloco. Consideramos que a manutenção do tecido tumoral em bloco é fundamental para que tração possa ser exercida sobre o tumor, e não sobre a medula, apresentando os vasos e aderências que cruzam essa interface para irrigar o tecido neoplásico e facilitando a sua divisão. Isso evita que os fascículos medulares sejam manipulados continuadamente durante o procedimento, o que é sempre desejável num tecido não complacente como a medula espinhal.

A monitorização eletrofisiológica durante o procedimento parece melhorar o resultado da ressecção dos ependimomas intramedulares, levando à ressecção total e reduzindo déficits pós-operatórios. ${ }^{14}$ Todas as séries utilizaram a monitorização de potenciais evocados somatossensoriais e motores para guiar a técnica microcirúrgica, o que não ocorreu em 14 dos 20 pacientes apresentados nesta série (70\%), por indisponibilidade rotineira de tal recurso no serviço.

Dos 20 pacientes tratados nesta série, somente um (5\%) não teve seu tumor ressecado completamente. $\mathrm{O}$ mesmo paciente foi o único com um ependimoma anaplásico e é o mais jovem da série, com 13 anos. A ressecção subtotal se deveu à dificuldade de se identificar $\mathrm{e}$ estabelecer o exato plano de separação entre o tumor e o tecido medular encontrado durante o procedimento. Apesar de haver questionamentos sobre a real existência de ependimomas infiltrativos da medula espinhal, ${ }^{3}$ esse caso de nossa série, bem como relatos de casos semelhantes nas séries citadas, ${ }^{10,11}$ chama a atenção para a possibilidade de se deparar com ependimoma infiltrativo, sem limites nítidos com a medula espinhal, com menor possibilidade de sucesso microcirúrgico, especialmente nos pacientes mais jovens, mesmo quando o aspecto na ressonância magnética for típico de ependimoma intramedular.

A técnica descrita neste trabalho teve $90 \%(18 / 20)$ de resultados satisfatórios, ou seja, ressecção total sem piora clínica (Tabela 1). A média de pontuação de McCormick no pré-operatório foi de 2,05, contra 1,85 no pós-operatório tardio. Tal diferença não foi significativa para o grupo como um todo $(\mathrm{p}>0,05)$. Ao segmentarmos a análise por grupo funcional no pré-operatório, a diferença não foi significativa para os pacientes grau III e IV. Não poderia haver melhora da média para os pacientes grau I, uma vez que esses pacientes possuem o melhor grau funcional existente. Mas o grupo de pacientes classificados como grau II no pré-operatório mostrou média de 1,57 no pós-operatório (contra óbvia média de 2,0 no pré-operatório), e essa diferença foi significativa, $\operatorname{com} \mathrm{p}=0,039(\mathrm{p}<0,05)$. Nenhum paciente com grau I piorou clinicamente e nenhum paciente grau IV apresentou melhora no seu status funcional. Conforme já confirmado na literatura, os pacientes em nossa série com graus I e II tiveram tendência a melhor resultado cirúrgico, não havendo nenhuma piora clínica nos pacientes desses grupos. Consideramos que quanto maior o grau na escala funcional de McCormick, menor a complacência medular à manipulação cirúrgica e maior a proximidade da superfície anterior do tumor a ramos primários e à própria artéria espinhal anterior, geralmente por causa do maior diâmetro axial das lesões. Epstein et al. ${ }^{3}$ afirmam que o manto medular circundando o tumor menor que $1 \mathrm{~mm}$ pela ressonância magnética pode significar pior resultado cirúrgico e ocorre mais frequentemente nos pacientes com grau de McCormick > II. Isso parece contribuir para uma menor chance de melhora clínica dos pacientes com graus III e IV no pré-operatório.

Dois pacientes submetidos à laminectomia da transição cervicotorácica apresentaram cifotização pós-operatória e necessitaram de artrodese do segmento tardiamente, nenhum paciente apresentou piora clínica e tinham idade de 31 e 37 anos no momento da primeira cirurgia. A cifotização é mais comum nos pacientes jovens, em especial crianças e adolescentes, principalmente na região cervical ou transição para coluna torácica. A laminotomia em open-door ou en bloc reduz a incidência de cifotização pós-operatória ${ }^{15} \mathrm{e}$ passou a ser a escolha do serviço para o acesso nos casos operados após 2005. A artrodese na primeira intervenção cirúrgica teria indicação para pacientes jovens, em transição cervicotorácica e que apresentem retificação ou cifotização da lordose cervical no pré-operatório, o que não ocorreu em nenhum dos casos desta série.

\section{Conclusão}

Os pacientes com melhores graus funcionais de McCormick (graus I e II) têm melhor evolução clínica 
e o tratamento microcirúrgico deve ser oferecido no momento do diagnóstico. A técnica microcirúrgica para retirada em bloco dessas lesões descrita neste trabalho é eficiente para promover a ressecção total das lesões e segura para não gerar déficits no pós-operatório tardio e perda no status funcional definitivo nos pacientes independentes funcionalmente antes da cirurgia.

\section{Conflito de interesses}

Não houve conflitos de interesse na realização deste trabalho.

\section{Referências}

1. Elsberg CA, Beer E. The operability of intramedullary tumors of the spinal cord. A report of two operations with remarks upon the extrusion of the spinal cord. Am J Med Sci. 1911;142:636-47.

2. Sciubba DM, Liang D, Kothbauer KF, Noggle JC, Jallo GI. The evolution of intramedullary spinal cord tumor surgery. Neurosurgery. 2009;65(Suppl 6):84-91.

3. Epstein FJ, Farmer JP, Freed D. Adult intramedullary spinal cord ependymomas: the result of surgery in 38 patients. $\mathrm{J}$ Neurosurg. 1993;79(2):204-9.

4. Fessler RG, Sekhar L. Atlas of neurosurgical techniques: spine and peripheral nerves. New York: Thieme; 2006.

5. Ogden AT, Schwartz TH, McCormick PC. Spinal cord tumors in adults. In: Winn RW. Youmans neurological surgery. 6th ed. Philadelphia: Saunders; 2011. p. 3131-43.

6. Woodworth GF, Chaichana KL, McGirt MJ, Sciubba DM, Jallo Gl, Gokaslan Z, et al. Predictors of ambulatory function after surgical resection of intramedullary spinal cord tumors. Neurosurgery. 2007;61(1):99-105.

7. Karikari IO, Nimjee SM, Hodges TR, Cutrell E, Hughes BD, Powers CJ, et al. Impact of tumor histology on resectability and neurological outcome in primary intramedullary spinal cord tumors: a single-center experience with 102 patients. Neurosurgery. 2011;68(1):188-97.

8. McCormick PC, Stein BM. Intramedullary tumors in adults. Neurosurg Clin N Am. 1990;1(3):609-30.

9. Hanbali F, Fourney DR, Marmor E, Suki D, Rhines LD, Weinberg JS, et al. Spinal cord ependymoma: radical surgical resection and outcome. Neurosurgery. 2002;51(5):1162-72.

10. Hoshimaru M, Koyama T, Hashimoto N, Kikuchi H. Results of microsurgical treatment for intramedullary spinal cord ependymomas: analysis of 36 cases. Neurosurgery. 1999;44(2):264-9.

11. Kucia EJ, Bambakidis NC, Chang SW, Spetzler RF. Surgical technique and outcomes in the treatment of spinal cord ependymomas, part 1: intramedullary ependymomas. Neurosurgery. 2011;68(Suppl Operative 1):57-63.

12. Aghakhani N, David P, Parker F, Lacroix C, Benoudiba F, Tadie M. Intramedullary spinal ependymomas: analysis of a consecutive series of 82 adult cases with particular attention to patients with no preoperative neurological deficit. Neurosurgery. 2008;62(6):1279-85.

13. Brotchi J, Fischer G. Spinal cord ependymomas. Neurosurg Focus. 1998;4(5):e2.

14. Sciubba DM, Chaichana KL, Woodworth GF, McGirt MJ, Gokaslan ZL, Jallo Gl. Factors associated with cervical instability requiring fusion after cervical laminectomy for intradural tumor resection. J Neurosurg Spine. 2008;8(5):413-9.

15. Boström A, von Lehe M, Hartmann W, Pietsch T, Feuss M, Boström JP, et al. Surgery for spinal cord ependymomas: outcome and prognostic factors. Neurosurgery. 2011;68(2):302-8.

\section{Endereço para correspondência}

Marcos Juliano dos Santos

Rua Sorocaba, 553, ap. 63, Vila Gatti

13300-340 - Itu, SP, Brasil

E-mail: mjs_neuro@yahoo.com.br 